\title{
Studying doubly charged Higgs pair production at the $\mathrm{LHC}$
}

\author{
Andi Hektor, Mario Kadastik, Kristjan Kannike, Mait Müntel, \\ and Martti Raidal
}

National Institute of Chemical Physics and Biophysics, Rävala 10, 10143 Tallinn, Estonia; andi.hektor@cern.ch

Received 21 February 2006

\begin{abstract}
The little Higgs models predict a rich phenomenology for the future collider experiments. Our attention is focused on the littlest Higgs model. We carry out a Monte Carlo study of the doubly charged Higgs pair production $\left(p p \rightarrow \Phi^{++} \Phi^{--}\right)$in a typical LHC experiment. The branching ratios are fixed using an assumption that, in addition, the observed masses of the neutrinos are generated by triplet Higgs: $\operatorname{BR}\left(\Phi^{ \pm \pm} \rightarrow \mu^{ \pm} \mu^{ \pm}\right)=$ $\operatorname{BR}\left(\Phi^{ \pm \pm} \rightarrow \mu^{ \pm} \tau^{ \pm}\right)=\operatorname{BR}\left(\Phi^{ \pm \pm} \rightarrow \tau^{ \pm} \tau^{ \pm}\right)=1 / 3$. We study the invariant mass distribution of same-charged muon pairs ( $\Phi^{ \pm \pm} \rightarrow 2 \mu^{ \pm \pm}$) together with the background processes from the standard model: $b \bar{b}, t \bar{t}$, and $Z Z$ production. To suppress the background, we propose a new type of selection rule, suitable in the case of production of the pairs of equal mass particles $\left(\Phi^{ \pm \pm}\right)$. This selection rule ensures high significance of the signal over the background of the standard model and implies very small cut of the signal under study. At the Monte Carlo level the doubly charged Higgs can be visible at the LHC in the mass range up to approximate $1 \mathrm{TeV}$.
\end{abstract}

Key words: little Higgs models, triplet Higgs, neutrino physics, LHC physics.

\section{INTRODUCTION}

The main motivation for the Large Hadron Collider (LHC) experiment is to reveal the secrets of electroweak symmetry breaking. Still, after the possible discovery of the standard model (SM) Higgs boson, a question will arise: what stabilizes Higgs mass against the Planck scale quadratically divergent radiative corrections? An answer to that question could be supersymmetry, which implies a very rich phenomenology of predicted s-particles in the future collider experiments. However, recently another possibility of formulating the physics of electroweak symmetry breaking, so-called the little Higgs, was proposed $\left[{ }^{1-3}\right]$. 
In the little Higgs models the SM Higgs boson is a pseudo Goldstone mode of a broken global symmetry and it remains light. It is much lighter than the other new modes of the model which have masses of order the symmetry breaking scale $\mathcal{O}(1) \mathrm{TeV}$. In order to cancel one-loop quadratic divergences to the SM Higgs mass, a new set of heavy gauge bosons $W^{\prime}, Z^{\prime}$ with the SM quantum numbers identical to $W, Z$, and a vector-like heavy quark pair $T, \bar{T}$ with charge $2 / 3$ must be introduced. Notice that those fields are put in by hand in order to construct a model with the required properties. However, the minimal model, so-called littlest Higgs model based on the $S U(5) / S O(5)$ global symmetry, has a firm prediction from the symmetry breaking pattern alone: the existence of another $\mathcal{O}(1) \mathrm{TeV}$ pseudo Goldstone boson $\Phi$ with the $S U(2)_{L} \times U(1)_{Y}$ quantum numbers $\Phi \sim(3,2)\left[{ }^{4}\right]$.

Another important and yet open question in particle physics is the mechanism for providing the masses of neutrinos. The discussions lasted many decades and were finalized with the experimental improvement of the neutrino oscillations at the beginning of this millennium $\left[{ }^{5-7}\right]$. Interestingly, the existence of triplet Higgs $\Phi$ might also be required to generate Majorana masses to the left-handed neutrinos $\left[{ }^{8}\right]$. Nonzero neutrino masses and the mixing of neutrinos is the only experimentally well verified signal about the unknown physics beyond the SM. In the triplet neutrino mass mechanism $\left[{ }^{9}\right]$, which we assume in this work, the neutrino mass matrix is generated via

$$
\left(m_{\nu}\right)_{i j}=\left(Y_{\Phi}\right)_{i j} v_{\Phi},
$$

where $\left(Y_{\Phi}\right)_{i j}$ are the Majorana Yukawa couplings of the triplet to the lepton generations $i, j=e, \mu, \tau$, which are described by the Lagrangian

$$
L=i \bar{\ell}_{L i}^{c} \tau_{2} Y_{\Phi}^{i j}(\tau \cdot \Phi) \ell_{L j}+\text { h.c. },
$$

and $v_{\Phi}$ is the effective vacuum expectation value of the neutral component of the triplet induced via the explicit coupling of $\Phi$ to the SM Higgs doublet $H$ as $\mu \Phi^{0} H^{0} H^{0}$. Here $\mu$ has dimension of mass.

In the concept of seesaw $\mu \sim M_{\Phi}$ and the smallness of neutrino masses is attributed to the very high scale of triplet mass $M_{\Phi}$ via the smallness of $v_{\Phi}=$ $\mu v^{2} / M_{\Phi}^{2}$, where $v=174 \mathrm{GeV}$. However, in the littlest Higgs model the triplet mass scale is $\mathcal{O}(1) \mathrm{TeV}$, which alone cannot suppress $v_{\Phi}$. Therefore in this model $\mu \ll M_{\Phi}$, which can be achieved, for example, via shining as shown by Ma et al. [ $\left.{ }^{10,11}\right]$. In that case $v_{\Phi} \sim \mathcal{O}(0.1) \mathrm{eV}$. We also remind that $v_{\Phi}$ contributes to the SM oblique corrections, and the precision data fit $\hat{T}<2 \times 10^{-4}\left[{ }^{12}\right]$ sets an upper bound $v_{\Phi} \leq 1.2 \mathrm{GeV}$ on that parameter.

The cross section of the single $\Phi^{ \pm \pm}$production via the $W W$ fusion process $\left[{ }^{13}\right]$ $q q \rightarrow q^{\prime} q^{\prime} \Phi^{ \pm \pm}$scales as $\sim v_{\Phi}^{2}$. In the context of the littlest Higgs model this process, followed by the decays $\Phi^{ \pm \pm} \rightarrow W^{ \pm} W^{ \pm}$, was studied in the papers $\left[{ }^{14-16}\right]$. The detailed ATLAS simulation of this channel shows $\left[{ }^{16}\right]$ that in order to observe $1 \mathrm{TeV} \Phi^{ \pm \pm}$, one must have $v_{\Phi}>29 \mathrm{GeV}$. This is in conflict 
with the precision physics bound $v_{\Phi} \leq 1.2 \mathrm{GeV}$ as well as with the neutrino data. Therefore the $W W$ fusion channel is not experimentally promising for the discovery of the doubly charged Higgs.

In this work we perform a Monte Carlo analysis of the Drell-Yan pair production $\left[{ }^{13,17}\right] p p \rightarrow \Phi^{++} \Phi^{--}$of the doubly charged Higgs boson followed by the leptonic decays $\Phi^{ \pm \pm} \rightarrow 2 \ell^{ \pm}$in a typical LHC experiment. We assume that neutrino masses come from the coupling to the triplet Higgs, which fixes the $\Phi^{ \pm \pm}$ leptonic branching ratios. Due to the smallness of $v_{\Phi}$, we can neglect the decays to $W W$. The advantages of this process are the following:

1. For all practical purposes the production cross section is well predicted; its independence on the model-dependent unknown parameters can be neglected.

2 . The decay $\Phi^{ \pm \pm} \rightarrow \ell^{ \pm} \ell^{ \pm}$is lepton number violating and allows reconstruction of $\Phi^{ \pm \pm}$invariant mass from the same charged leptons rendering the SM background very small in the signal region. Therefore it should provide a very clean detectable signature for the LHC detectors.

3. The known neutrino mixing Eq. (1) predicts the branching ratios as $\operatorname{BR}\left(\Phi^{ \pm \pm} \rightarrow\right.$ $\left.\mu^{ \pm} \mu^{ \pm}\right)=\operatorname{BR}\left(\Phi^{ \pm \pm} \rightarrow \tau^{ \pm} \tau^{ \pm}\right)=\operatorname{BR}\left(\Phi^{ \pm \pm} \rightarrow \mu^{ \pm} \tau^{ \pm}\right)=1 / 3$. Here we assume that neutrinos have a normal hierarchy, which implies negligible decay rates to the electron final states.

In the current study we consider only the muon final states, which are the easiest to observe at the LHC environment. For example, the Compact Muon Solenoid (CMS) is optimized for muon signals and the discovery rate of energetic muons ( $p_{T}>2 \mathrm{GeV}$ and $|\eta|<2.4$, where $p_{T}$ is transfer momentum) is close to $95 \%\left[{ }^{18}\right]$.

We have generated the production process and the leptonic decays of $\Phi^{ \pm \pm}$ as well as the relevant background processes using the PYTHIA Monte Carlo generator $\left.{ }^{19}\right]$. We focus our study on the $2 \mu^{+} 2 \mu^{-}$final states as it is a very characteristic signature compared to the SM signatures. We use the default set of parameters (parton structure functions, gauge couplings, etc.) from CMS CMKIN $\left[{ }^{18}\right]$, except that we fix the $\Phi^{ \pm \pm}$branching ratios via $Y_{\Phi}^{\mu \mu}=\sqrt{2} Y_{\Phi}^{\mu \tau}=$ $Y_{\Phi}^{\tau \tau}=0.01$. Rescaling those couplings to satisfy data from the searches for lepton flavour violating processes $\left[{ }^{13,20}\right]$ does not affect our results. We also comment on the results of our analyses if this assumption is relaxed and $\operatorname{BR}\left(\Phi^{ \pm \pm} \rightarrow\right.$ $\left.\mu^{ \pm} \mu^{ \pm}\right)=1$

\section{SELECTION CRITERIA FOR THE $2 \mu^{+} 2 \mu^{-}$SIGNATURE}

Considering the four muon final states $2 \mu^{+} 2 \mu^{-}$as the experimental signature for the process $p p \rightarrow \Phi^{++} \Phi^{--}$, we have reconstructed the invariant mass of two like-sign muons,

$$
\left(m_{I}^{ \pm \pm}\right)^{2}=\left(p_{1}^{ \pm}+p_{2}^{ \pm}\right)^{2},
$$

with the four-momentas $p_{1,2}$ of muons. Since the like-sign signal muons originate from the same doubly charged Higgs boson, the invariant mass peak will measure the Higgs mass, $m_{I}=M_{\Phi}$. The four-muon signature is the cleanest and the most 
robust one. In addition, as the doubly charged Higgs is produced in pairs, we can use some unique selection techniques described below.

The background arises mostly from the $Z Z, b \bar{b}$, and $t \bar{t}$ production and their muonic decay. Because those particles are lighter than $\Phi$ (the present bound from Tevatron is $M_{\Phi} \geq 136 \mathrm{GeV}\left[{ }^{21,22}\right]$ ), the background muons must have smaller $p_{T}$ and it should not give an invariant mass peak. In addition, a self-generated low background process exists: if the branching ratio allows $\Phi^{ \pm \pm} \rightarrow \tau^{ \pm} \tau^{ \pm}$decay, then we get some soft energy muons from the $\tau$ decay (the branching ratio $\tau \rightarrow \mu$ is approximately $20 \%$ ).

We have applied four selection rules for our Monte Carlo generated particle data:

S1: Only the muons with pseudorapidity $|\eta|<2.4$ are detectable in the typical LHC detector, for example CMS $\left[{ }^{18}\right]$. Therefore, only the particles with $|\eta|<2.4$ are selected from our Monte Carlo generation.

S2: All muons with transfer momentum $p_{T}<25 \mathrm{GeV}$ are neglected.

S3: Only the events with at least two positive and two negative muons, so-called $2 \mu^{+} 2 \mu^{-}$events, are selected. The charge misidentification rate is very low for the LHC detectors as mentioned above.

S4: We require that both invariant masses in the pair production event be approximately equal. For this selection rule we have considered two different values of the cuts: (1) a wider one, $0.5<m_{I}^{++} / m_{I_{+}}^{--}<1.5$ and (2) a more restrictive one, $0.9<m_{I}^{++} / m_{I}^{--}<1.1$, where $m_{I}^{ \pm \pm}$is the invariant mass of the same charged muons.

The selection rule $\mathrm{S} 1$ is applied first as it is a natural restriction of the real detectors at the LHC. The restriction of the detectors suppresses mainly the soft energy background as it is more boosted. The $\mathrm{S} 2$ filter rules out all soft background particles and saves computational resources for the next steps of the analyses. The S3 rule is natural if we are looking for two doubly charged Higgs bosons with the opposite charges and we have a high charge identification rate (0.95). The final rule, $\mathrm{S} 4$, is based on the fact that the invariant masses of the same charged muon pairs have to be equal in the case of pair production of doubly charged Higgs boson. Naturally, some freedom is needed in the last condition due to decay width and experimental error of the detector. Therefore, we applied the two versions of the rule $\mathrm{S} 4$, marked below as $\mathrm{S} 4_{1}$ and $\mathrm{S} 4_{2}$.

\section{MONTE CARLO ANALYSES}

We have generated with PYTHIA Monte Carlo the data-sets of $2.8 \times 10^{7}$ $b \bar{b}, t \bar{t}$ and $10^{6} Z Z$ events for the background, and the data-sets of $5 \times 10^{5}$ signal events with $M_{\Phi}=200,500,1000 \mathrm{GeV}$. We have applied the selection rules described above and rescaled the results taking into account the cross section of the particular process. The cross sections have been taken from the paper $\left[{ }^{19}\right]$. In Table 1 we present the expected number of background and signal events, as well 
as the numbers of $\Phi^{ \pm \pm}$candidates $\left(2 \mu^{+} 2 \mu^{-}\right.$events) in total and in the detector $(|\eta|<2.4)$. We assume the total integrated luminosity of the LHC to be $300 \mathrm{fb}^{-1}$.

Table 2 describes the effect of the different selection rules. As one can see, after the last selection S4 the background is almost eliminated around the invariant mass peaks. In Table 3 we present the final significances of the Higgs signal and

Table 1. The number of expected background and signal events for the integral luminosity of $300 \mathrm{fb}^{-1}$. In case of Higgs events a self-generated background with $2 \mu^{+} 2 \mu^{-}$final state exists, which originates from secondary tau decay to muons. So, the primary $2 \mu^{+} 2 \mu^{-}$events are given in brackets. For the signal events we have taken the following branching ratios: $\operatorname{BR}\left(\Phi^{ \pm \pm} \rightarrow \mu^{ \pm} \mu^{ \pm}\right)=\operatorname{BR}\left(\Phi^{ \pm \pm} \rightarrow \mu^{ \pm} \tau^{ \pm}\right)=\operatorname{BR}\left(\Phi^{ \pm \pm} \rightarrow \tau^{ \pm} \tau^{ \pm}\right)=1 / 3$

\begin{tabular}{l|c|c|c}
\hline \multicolumn{1}{c|}{ Process } & $\begin{array}{c}\mathrm{N} \text { of } 4 \ell \\
\text { events }\end{array}$ & $\begin{array}{c}\mathrm{N} \text { of } 2 \mu^{+} 2 \mu^{-} \\
\text {events }\end{array}$ & $\begin{array}{c}\mathrm{N} \text { of } 2 \mu^{+} 2 \mu^{-} \text {events } \\
\text { in detector }(\mathrm{S} 1:|\eta|<2.4)\end{array}$ \\
\hline Background & \multicolumn{3}{c}{} \\
$t \bar{t}$ & $2.6 \times 10^{7}$ & $4.6 \times 10^{5}$ & $3.0 \times 10^{5}$ \\
$b \bar{b}$ & $2.8 \times 10^{10}$ & $2.5 \times 10^{7}$ & $9.7 \times 10^{6}$ \\
$Z Z$ & $2.1 \times 10^{4}$ & $5.8 \times 10^{3}$ & $2.3 \times 10^{3}$ \\
Signal & & & \\
$M_{\Phi}=200 \mathrm{GeV}$ & 23354 & $4045(2593)$ & $2416(1797)$ \\
$M_{\Phi}=500 \mathrm{GeV}$ & 596.4 & $102(66.0)$ & $75.6(55.8)$ \\
$M_{\Phi}=1000 \mathrm{GeV}$ & 16.75 & $2.9(1.87)$ & $2.26(1.68)$
\end{tabular}

Table 2. The number of $\Phi^{ \pm \pm}$candidates form $2 \mu^{+} 2 \mu^{-}$final states passing all the selection rules. The primary $2 \mu^{+} 2 \mu^{-}$events are given in brackets. For the signal the branching ratios are $\mathrm{BR}\left(\Phi^{ \pm \pm} \rightarrow \mu^{ \pm} \mu^{ \pm}\right)=\mathrm{BR}\left(\Phi^{ \pm \pm} \rightarrow \mu^{ \pm} \tau^{ \pm}\right)=\mathrm{BR}\left(\Phi^{ \pm \pm} \rightarrow \tau^{ \pm} \tau^{ \pm}\right)=1 / 3$

\begin{tabular}{l|llll}
\hline \multirow{2}{*}{ Process } & \multicolumn{5}{c}{ Number of invariant masses } \\
\cline { 2 - 5 } & \multicolumn{1}{c}{ No cut } & \multicolumn{1}{c}{$p_{T}>25$} & \multicolumn{1}{c}{$\mathrm{S} 4_{1}$} & \multicolumn{1}{c}{$\mathrm{S} 4_{2}$} \\
\hline Energy range $100-300 \mathrm{GeV}$ & & & \\
$t \bar{t} \rightarrow 2 \mu^{+} 2 \mu^{-}$ & 17192 & 875 & 381 & 86 \\
$b \bar{b} \rightarrow 2 \mu^{+} 2 \mu^{-}$ & 20196 & 1009 & 0 & 0 \\
$Z Z \rightarrow 2 \mu^{+} 2 \mu^{-}$ & 1313 & 655 & 345 & 67 \\
$M_{\Phi}=200 \mathrm{GeV}$ & $4831(3593)$ & $3909(3212)$ & $3664(3178)$ & $3182(2863)$ \\
Energy range $250-750 \mathrm{GeV}$ & & & \\
$t \bar{t} \rightarrow 2 \mu^{+} 2 \mu^{-}$ & 754 & 76.6 & 13.6 & 0 \\
$b \bar{b} \rightarrow 2 \mu^{+} 2 \mu^{-}$ & 0 & 0 & 0 & 0 \\
$Z Z \rightarrow 2 \mu^{+} 2 \mu^{-}$ & 172 & 81.5 & 26.6 & 3.0 \\
$M_{\Phi}=500 \mathrm{GeV}$ & $151(112)$ & $143(111)$ & $129(109)$ & $98(96)$ \\
Energy range $500-1500 \mathrm{GeV}$ & & & \\
$t \bar{t} \rightarrow 2 \mu^{+} 2 \mu^{-}$ & 18.4 & 1.9 & 0 & 0 \\
$b \bar{b} \rightarrow 2 \mu^{+} 2 \mu^{-}$ & 0 & 0 & 0 & 0 \\
$Z Z \rightarrow 2 \mu^{+} 2 \mu^{-}$ & 21.7 & 10.6 & 1.7 & 0 \\
$M_{\Phi}=1000 \mathrm{GeV}$ & $4.52(3.37)$ & $4.37(3.36)$ & $3.87(3.30)$ & $2.91(2.88)$
\end{tabular}


Table 3. The significance and the percentage of invariant masses left after the selection process. The primary $2 \mu^{+} 2 \mu^{-}$events are given in brackets. For the signal the branching ratios are $\mathrm{BR}\left(\Phi^{ \pm \pm} \rightarrow \mu^{ \pm} \mu^{ \pm}\right)=\mathrm{BR}\left(\Phi^{ \pm \pm} \rightarrow \mu^{ \pm} \tau^{ \pm}\right)=\mathrm{BR}\left(\Phi^{ \pm \pm} \rightarrow \tau^{ \pm} \tau^{ \pm}\right)=1 / 3 . \quad$ N/A - not available

\begin{tabular}{|c|c|c|c|c|c|c|}
\hline \multirow[t]{2}{*}{ Process } & \multicolumn{3}{|c|}{ Significance } & \multicolumn{3}{|c|}{ Signal left after the selection, \% } \\
\hline & $p_{T}>25$ & $\mathrm{~S} 4_{1}$ & $\mathrm{~S} 4_{2}$ & $p_{T}>25$ & $\mathrm{~S} 4_{1}$ & $\mathrm{~S} 4_{2}$ \\
\hline Total background & & N/A & & 0.036 & 0.011 & 0.002 \\
\hline$M_{\Phi}=200 \mathrm{GeV}$ & 77.5 & 135.9 & 257.0 & $80.9(89.3)$ & $75.8(88.4)$ & $65.8(79.6)$ \\
\hline$M_{\Phi}=500 \mathrm{GeV}$ & 11.3 & 20.4 & 57.0 & $94.4(98.9)$ & $85.5(97.2)$ & $64.8(85.9)$ \\
\hline$M_{\Phi}=1000 \mathrm{GeV}$ & 1.2 & 2.9 & $\infty$ & $96.6(99.7)$ & $85.6(97.8)$ & $64.3(85.4)$ \\
\hline
\end{tabular}

the final lefts of the background and Higgs signal after all the selection filters. The significance is defined as $S / \sqrt{B}$, where $S$ and $B$ are the numbers of the signal and background events. Figure 1 plots the histogram for the invariant mass distribution of the like-sign muons for $M_{\Phi}=500 \mathrm{GeV}$ and $M_{\Phi}=1000 \mathrm{GeV}$. The darkest histogram marks the signal left after the full selection and the lightest histogram marks the signal in the detector $(|\eta|<2.4)$. For those values of $M_{\Phi}$ the significance is huge.

For the mass $M_{\Phi}=1 \mathrm{TeV}$ one expects only $\sim 3$ doubly charged Higgs candidates, although the total number of produced $\Phi^{ \pm \pm}$is $\sim 33$. As $\operatorname{BR}\left(\Phi^{ \pm \pm} \rightarrow\right.$ $\left.\mu^{ \pm} \mu^{ \pm}\right)=1 / 3$, the strong signal suppression occurs because the probability for both $\Phi^{ \pm \pm}$to decay to two muons is $(1 / 3)^{2}=1 / 9$. So, the probability to produce two or more Higgs pair events is about $40 \%$. Thus, the LHC can reach the $\Phi$ mass close to $1 \mathrm{TeV}$. However, if $\operatorname{BR}\left(\Phi^{ \pm \pm} \rightarrow \mu^{ \pm} \mu^{ \pm}\right)=1$, we expect to get $\sim 26$ Higgs candidates for $M_{\Phi}=1 \mathrm{TeV}$. In this case the LHC mass reach extends up to $1.2 \mathrm{TeV}$.

\section{CONCLUSIONS}

We have carried out Monte Carlo study of doubly charged Higgs boson pair production, followed by leptonic decays at the LHC experiments. Since the single $\Phi^{ \pm \pm}$production is strongly suppressed, this is the only potentially observable channel at the LHC. In addition, we have assumed that triplet Higgs also generates the observed neutrino masses, which fixes the $\Phi^{ \pm \pm}$leptonic decay branching ratios from neutrino data. We have generated the signal as well as the background processes for four muon final states with PYTHIA Monte Carlo, and analysed how to reduce maximally the SM background to reach maximum significance of the signal. Our results are plotted in Fig. 1, which show that the invariant mass distribution of the like-sign muon pairs allows discovery of the doubly charged Higgs with the mass close to $M_{\Phi}=1 \mathrm{TeV}$. Relaxing our assumption about branching ratios, and assuming $\operatorname{BR}\left(\Phi^{ \pm \pm} \rightarrow \mu^{ \pm} \mu^{ \pm}\right)=1$, the LHC discovery reach for $\Phi^{ \pm \pm}$increases to $M_{\Phi}=1.2 \mathrm{TeV}$ 

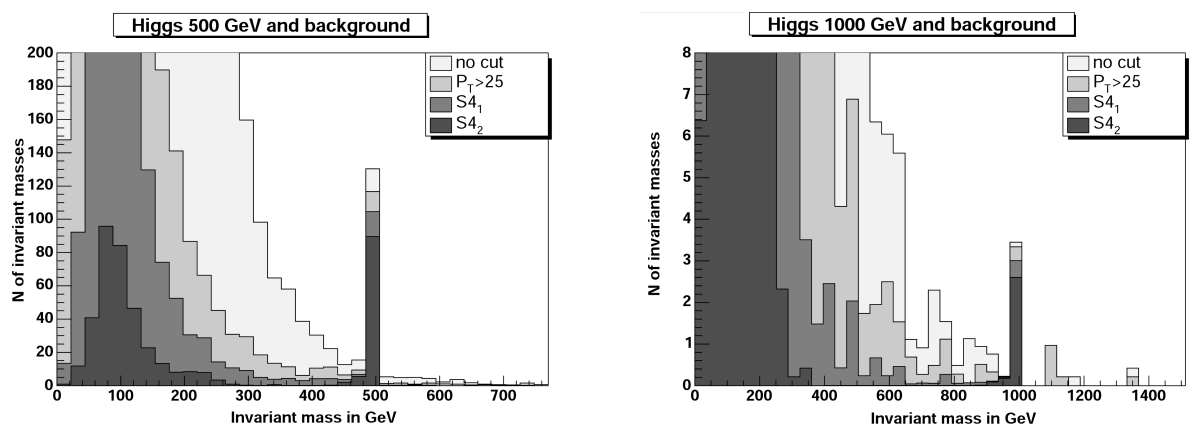

Fig. 1. Distribution of the invariant mass of two like-sign muons. The darkest histogram marks the signal after the full set of cuts for the $2 \mu^{+} 2 \mu^{-}$final state in the case of $M_{\Phi}=500 \mathrm{GeV}$ and $M_{\Phi}=1000 \mathrm{GeV}$ (the left and the right panel, respectively). The background is very low around the invariant mass peak of the Higgs signal. In addition to the standard model background, the small "tail" of the self-generated $\Phi \rightarrow \tau \rightarrow \mu$ background is visible at the smaller invariant mass region close to the Higgs mass peak. The lighter histograms mark the signal with less selections applied, as indicated in the figures.

Our results can be improved by including the tau-lepton reconstruction in the analyses. Alternatively, the background can further be reduced using some standard methods: vetoing the b-tagged events, by reconstructing $Z$ and $t \bar{t}$, and neglecting leptons from their decays. In addition, the pair production gives some additional possibilities of constructing new selection rules. Nevertheless, since the signal is so robust and clean, our results show that this is not necessary for the region up to $M_{\Phi}<1 \mathrm{TeV}$.

\section{ACKNOWLEDGEMENTS}

We thank S. Lehti, A. Nikitenko, and A. Strumia for useful discussions and technical advice. This work is partially supported by grant No. 6140 of the Estonian Science Foundation, by EC I3 contract No. 026715, and by the Estonian Ministry of Education and Research. The calculations are performed in the Estonian Grid $\left.{ }^{23}\right]$ and Baltic Grid.

\section{REFERENCES}

1. Arkani-Hamed, N., Cohen, A. G. and Georgi, H. (De)constructing dimensions. Phys. Rev. Lett., 2001, 86, 4757-4761.

2. Cheng, H.-C., Hill, C. T., Pokorski, S. and Wang, J. The standard model in the latticized bulk. Phys. Rev., 2001, D64, 065007.

3. Arkani-Hamed, N., Cohen, A. G. and Georgi, H. Electroweak symmetry breaking from dimensional deconstruction. Phys. Lett., 2001, B513, 232-240. 
4. Arkani-Hamed, N., Cohen, A. G., Katz, E. and Nelson, A. E. The littlest Higgs. JHEP, 2002, 07, 034.

5. Ashie, Y. et al. A measurement of atmospheric neutrino oscillation parameters by superkamiokande i. Phys. Rev., 2005, D71, 112005.

6. Aliu, E. et al. Evidence for muon neutrino oscillation in an accelerator-based experiment. Phys. Rev. Lett., 2005, 94, 081802.

7. Ahmed, S. N. et al. Measurement of the total active ${ }^{8}$ B solar neutrino flux at the Sudbury Neutrino Observatory with enhanced neutral current sensitivity. Phys. Rev. Lett., 2004, 92, 181301.

8. Schechter, J. and Valle, J. W. F. Neutrino masses in $\mathrm{SU}(2) \times \mathrm{U}(1)$ theories. Phys. Rev., 1980, D22, 2227-2235.

9. Ma, E. and Sarkar, U. Neutrino masses and leptogenesis with heavy Higgs triplets. Phys. Rev. Lett., 1998, 80, 5716-5719.

10. Ma, E., Raidal, M. and Sarkar, U. Verifiable model of neutrino masses from large extra dimensions. Phys. Rev. Lett., 2000, 85, 3769-3772.

11. Ma, E., Raidal, M. and Sarkar, U. Phenomenology of the neutrino-mass-giving Higgs triplet and the low-energy seesaw violation of lepton number. Nucl. Phys., 2001, B615, 313-330.

12. Marandella, G., Schappacher, C. and Strumia, A. Little-Higgs corrections to precision data after CERN LEP2. Phys. Rev., 2005, D72, 035014.

13. Huitu, K., Maalampi, J., Pietila, A. and Raidal, M. Doubly charged Higgs at LHC. Nucl. Phys., 1997, B487, 27-42.

14. Han, T., Logan, H. E., McElrath, B. and Wang, L.-T. Phenomenology of the little Higgs model. Phys. Rev., 2003, D67, 095004.

15. Han, T., Logan, H. E. and Wang, L.-T. 2005 Smoking-gun signatures of little Higgs models. http://arxiv.org/abs/hep-ph/0506313

16. Azuelos, G. et al. Exploring little Higgs models with atlas at the LHC. Eur. Phys. J., 2005, C39S2, 13-24.

17. Gunion, J. F., Grifols, J., Mendez, A., Kayser, B. and Olness, F. I. Higgs bosons in leftright symmetric models. Phys. Rev., 1989, D40, 1546.

18. Della Negra, M. et al. CMS, the Muon Project: Technical Design Report. CERN, 1997.

19. Sjostrand, T. et al. High-energy-physics event generation with PYTHIA 6.1. Comput. Phys. Commun., 2001, 135, 238-259.

20. Barenboim, G., Huitu, K., Maalampi, J. and Raidal, M. Constraints on doubly charged Higgs interactions at linear collider. Phys. Lett., 1997, B394, 132-138.

21. Acosta, D. et al. Search for doubly-charged Higgs bosons decaying to dileptons in $p \bar{p}$ collisions at $\sqrt{s}=1.96$ TeV. Phys. Rev. Lett., 2004, 93, 221802.

22. Abazov, V. M. et al. Search for doubly-charged Higgs boson pair production in the decay to $\mu^{+} \mu^{+} \mu^{-} \mu^{-}$in $p \bar{p}$ collisions at $\sqrt{s}=1.96$ TeV. Phys. Rev. Lett., 2004, 93, 141801.

23. Hektor, A., Anton, L., Kadastik, M., Skaburskas, K. and Teder, H. The first scientific results from the Estonian Grid. Proc. Estonian Acad. Sci. Phys. Math., 2004, 54, 111127.

\section{Topeltlaetud Higgsi bosoni paarissignaali uurimine LHC-eksperimendi juures}

\section{Andi Hektor, Mario Kadastik, Kristjan Kannike, Mait Müntel ja Martti Raidal}

Nn väikesed Higgsi mudelid lubavad mitmekesist osakeste fenomenoloogiat tulevaste kiirendieksperimentide jaoks. Artiklis on uuritud võimalikke eksperi- 
mentaalseid märke nn väikseima Higgsi mudeli poolt ennustatud nähtustest skaalal 0,2 kuni $1 \mathrm{TeV}$. Võimalike signatuuride uurimiseks on kasutatud meie poolt PYTHIA Monte Carlo generaatoriga toodetud andmeid, mis vastavad topeltlaetud Higgsi bosoni paaridele tüüpilise LHC-eksperimendi jaoks (prootonite kokkuporrge masskeskme energiaga $\sim 14 \mathrm{TeV}$, integraalne luminositeet $300 \mathrm{fb}^{-1}$ ). Selleks on fikseeritud topeltlaetud Higgsi erinevate lagunemiskanalite tõenäosused, kasutades meie poolt pakutud seost väikseima Higgsi mudeli ja neutriinode massimaatriksi vahel. Signatuure on hinnatud samamärgiliselt laetud müüonite paaride invariantsete masside põhjal ja seda koos standardmudelist pärit taustaprotsessidega. Kuna tegemist on topeltlaetud Higgsi paaride tekkimisega, siis saab taustaprotsesside mahasurumiseks rakendada mõningaid unikaalseid meetodeid. Tänu sellele võib signaali statistilist olulisust tunduvalt tõsta ja signatuuri on võimalik muuta nähtavaks kuni piirkonnani, kui Higgsi mass läheneb $1 \mathrm{TeV}$-ile. 